\title{
Comparison of high-field and low-field magnetic resonance imaging of stifle joint disorders in dogs
}

\author{
A. Przeworski, Z. Adamiak, J. Gtodek \\ Faculty of Veterinary Medicine, University of Warmia and Mazury, Department of Surgery and Radiology, \\ Oczapowskiego 14, 10-957 Olsztyn, Poland
}

\begin{abstract}
The most common cause of hindlimb lameness in dogs is cranial cruciate ligament rupture. In $48-77.3 \%$ of the population this trauma leads to secondary damage of the meniscus. Depending on the magnetic strength of the used device, different diagnostic accuracy can be achieved. The examination sensitivity of magnetic resonance imaging is affected by many factors which are independent of diagnostic strength, such as correct positioning of the patient, size of the stifle joint examined, or selection of the right protocol of sequences. Sensitivity of meniscus damage detection was $100 \%$ and $90 \%$, respectively, in high- and low-field magnetic resonance. The best results were reported during examination of the stifle in dogs above $10 \mathrm{~kg}$ b.w. at a flexion angle of $145^{\circ}$, and in sagittal and dorsal planes. Regardless of the magnetic strength applied, imaging of the whole cranial cruciate ligament is difficult. Moreover, MRI allows the detection of the first signs of osteoarthritis, which were observed 4 and 6 weeks after rupture of the cranial cruciate ligament using high and low-field MRI. This also applies to lesions in the subchondral bone or a bone marrow which occurred in association with insufficiency of the stifle joint, and were mainly localized in the epiphysis of the femur and tibia. The present article provides a comparison of different examination protocols and images of damaged stifle structures, such as menisci, ligaments and bones of the stifle joint visualized with low-field and high-field magnetic resonance. Magnetic resonance arthrography is also discussed.
\end{abstract}

Key words: magnetic resonance imaging, canine, stifle joint, cranial cruciate ligament, meniscus, arthrography, tears

\section{Introduction}

Historically, the magnetic field gradient was used in the 1970s to perform the diagnostic imaging (Macchia et al. 2007). It was a new way of imaging characterized by better contrast resolution of a soft tissue in comparison with traditional radiography (Baird et al. 1998a). Since that time, there have been more devices with higher magnetic fields, starting from low-field, through midfield, to high-filed. The increased quality of the images obtained using low-field devices and the cost of receiving them achieved in the mid-to-late 1990s renewed interest in low magnetic fields (Rutt and Lee 1996, Gavin 2011). 
It is assumed that low-field magnetic resonance has a field strength of between 0.2T and 0.4T (Konar and Lang 2011). Other authors reported less than $0.15 \mathrm{~T}$ (Baird et al. 1998a) or 0.15T to 0.3T (Sabiston et al. 1987, Martig et al. 2007). Whereas high-field magnetic resonance has a magnetic strength of 1 to 1.5T (Nolte-Ernsting et al. 1996, Libicher et al. 2005) or higher.

Magnetic resonance imaging (MRI) has been particularly used in the visualization of lesions in the musculoskeletal system, spine, or in intracranial changes (as cited in Konar and Lang 2011, Adamiak et al. 2011). MRI has become the preferred noninvasive imaging modality for the evaluation of normal stifle joints. It is also a particularly useful diagnostic tool for stifle injuries and secondary changes (Widmer et al. 1991, Conaghan et al. 2006, D'Anjou et al. 2008, Pujol et al. 2011, Tremolada et al. 2014) Among these changes, lesions of cruciate ligaments, menisci, synovitis, cartilage lesions, osteoarthritis and neoplastic tumors stand out.

Moreover, the stifle joint can be imaged using ultrasonography, conventional or digital radiography, or computed tomography (CT). A sonographic study is useful for imaging changes such structures as ligaments, muscles, tendon cartilages, and meniscal tears (Reed et al. 1995), although clinical interpretation of meniscal abnormalities is difficult (Barrett et al. 2009). Computed tomography also allows distinguishing of all structures of the stifle with diagnostic accuracy.

The purpose of this article is to present the current knowledge regarding the imaging of the canine stifle joint with low and high field magnetic resonance imaging.

\section{Magnetic resonance imaging of stifle joint}

The MRI image quality is affected not only by field strength (Parizel et al. 1995, Konar and Lang 2011). Gradient strength and slew rate, the coil, as well as the homogeneity of the magnetic field are also important parameters. The skills of the operator are also invaluable. The MRI coil has a significant impact on the signal-to-noise ratio, and thus on graininess (Mink et al. 1993, Banfield and Morrison 2000). Therefore, it is advisable to use a coil well-adjusted to the size of the stifle, which allows for better visibility of the cranial cruciate ligament (Podadera et al. 2014). Worthy of mention is a study in human medicine during which the sensitivity of the detection of meniscal tears in the knee with a standard knee coil and a surface coil were compared, and it amounted to $76 \%$ and $88 \%$, respectively (Nemec et al. 2008). While
Banfield and Morrison (2000) used a wrist coil and received satisfactory results.

The authors emphasized the position of the dog as an important factor in the imaging of intraarticular structures. Among them the following may be mentioned: dorsal recumbency with extended (Baird et al. 1999, Martig et al. 2006) or flexed hindlimbs (Bottcher et al. 2010, Tremolada et al. 2014), lateral recumbency with various flexion angles of the stifle (Barrett et al. 2009, Podadera et al. 2014), or even in sternal recumbency (Harper et al. 2011). Pujol et al. (2011) reported that lateral recumbency with the stifle flexed at $145^{\circ}$ changed the tension of the cranial cruciate ligament and improved its visibility. Whereas Tremolada et al. (2014) used dorsal recumbency because various angles of stifle flexion using a special device were easier to obtain. Among other things, this factor was focused on by McCartney and McGovern (2012), who defined lateral recumbency with the examined limb on top as the best way to visualize menisci. Furthermore, dorsal recumbency was also suitable, but the technique was associated with some problems of positioning within the coil.

\section{Comparison of high-field magnetic resonance imaging and low-field magnetic resonance imaging}

\section{Ligaments}

Cranial cruciate ligament injuries are the most common orthopedic diseases in dogs (Simpler et al. 2014). Although it is assumed that the problems associated with the cranial cruciate ligament occur in large or giant breed dogs, it is true that they can affect any dog (Hayashi et al. 2004, Podadera et al. 2014), especially neutered dogs (Witsberger et al. 2008). On MRI images, undamaged caudal and cranial ligaments are uniform low signal intensity bands of oblique course, which makes it difficult to image them entirely on one slice. Therefore, a study was performed to determine the optimal stifle flexion angle to improve the visibility of ligaments. Podadera et al. (2014) compared various flexion angles such as $90^{\circ}$, $135^{\circ}, 145^{\circ}$ using low-field MRI. They reported that the cranial cruciate ligament was best visualized at $90^{\circ}$. Slightly more often it is possible to see the caudal cruciate ligament in its entirety (Pujol et al. 2011).

There are only a few studies on imaging of damaged cruciate ligaments using MRI. There is particular interest in secondary changes to partial or total ruptured cranial cruciate ligaments. Furthermore, the diagnosis of partial rupture of the cranial cruciate ligament is also difficult in human medicine (Tsai et 
al. 2004, Barrett et al. 2009). The specific signs characterize anterior cruciate ligament complete rupture, such as discontinuity, signal changes, and location other than normal (Brandser et al. 1996), while the partial rupture has an increased signal within the ligament without signs of discontinuity (Van Dyck et al. 2012).

In addition, in human beings acute and chronic tears of ligaments were distinguished. Acute tears in magnetic resonance images were characterized by edema of soft tissues, as well as diffuse or focal changes of visibility of the ligament. Chronic tears were characterized by more varied changes, which sometimes allowed for receiving an image mimicking the entire ligament due to the presence of scarred fragments (Vahey et al. 1991, Tsai et al. 2004, Barrett et al. 2009). Barrett et al. (2009) received an image of increased signal intensity in the ligament, which probably indicated the presence of chronic tears. The study presented above was performed using high-field MRI. Moreover, it was observed that fascia lata used in surgical treatment could produce hypointense images, which erroneously resembled the entire cranial cruciate ligament (Barrett et al. 2009).

Generally, the cruciate ligaments are evaluated in the sagittal plane, in which diagnostic quality was good (Banfield and Morrison 2000). Barrett et al. (2009), with high-field MRI, used proton density-weighted (PD-weighted) images in the sagittal plane to evaluate cranial cruciate ligament abnormalities with a sensitivity of 0.93 . In addition, in order to improve visibility oblique scans were performed that were aligned to the cranial cruciate ligament regardless of the strength of the magnetic field (Baird et al. 1998a, Barrett et al. 2009). Other authors used T2-weighted fast spin echo (FSE) sequence due to the difference in signal intensity between the cranial cruciate ligament and synovial fluid. Furthermore, it avoids the risk of magic angle artifact (Podadera et al. 2014). Besides that, the T2-weighted FSE and PD-weighted with fat-suppressed sequences were the best to visualize joint effusion, edema and inflammation of stifle joint (Bredella et al. 2000, Blond et al. 2008, D'Anjou et al. 2008). In another study, the diagnosis of a cranial cruciate ligament rupture, even by means of a 3T, was difficult, and was characterized by an increase in the signal intensity of a hypointense ligament (Galindo-Zamora et al. 2013).

The caudal cruciate ligament injury occurs less frequently. Lesions of the caudal cruciate ligament which were histological changes not visible intraoperatively were observed using a high magnetic field hyperintense (Galindo-Zamora et al. 2013).

The damage to the cranial cruciate ligament is also the most common cause of canine stifle osteoar- thritis (Carrig 1997, D'Anjou et al. 2008). One of the difficulties encountered in imaging is the thickness of the stifle articular cartilage, which is between 0.6-1.3 $\mathrm{mm}$ (Pujol et al. 2011). Cross-section images obtained by magnetic resonance imaging is an excellent tool to recognize early osteophytes (Carrig 1997, D'Anjou et al. 2008). Baird et al. (1999) used low-field magnetic resonance to visualize degenerative changes, which were characterized by an increased signal intensity weight-bearing portion of the articular cartilage in T1-weight sequence 6 weeks from the transection of the cranial cruciate ligament. It was probably an early sign of osteoarthritis. T2-wieghted, and proton density-weighted sequences were not useful to the assessment of articular cartilage changes. Furthermore, the articular cartilage with synovial effusion on T2-weighted sequences was difficult to identify using low-field MRI, while other results are presented in human medicine research where effusion increased the contrast between various structures of the stifle joint, but these images were performed using high-field MRI (Baird et al. 1999). Similar results were reported by D'Anjou et al. (2008), where high-field MRI allowed the imaging of a greater amount of osteophytes than by means of computed radiography. The osteophytes were observed as early as 4 weeks after transection of the cranial cruciate ligament. They have high signal intensity which contrast well with a hypointense image of the bone cortex. Moreover, D'Anjou et al. (2008) used the T1-weighted gradient echo (GE) without fat saturation sequences that visualize pathologic changes of the osseous tissue due to a significant increase in signal intensity of the bone marrow and articular soft tissue structures compared to the hypointense cortical bone. The detection of early degenerative changes in magnetic resonance is becoming the perfect utility in the evaluation of the effectiveness of drugs such as disease-modifying osteoarthritis drugs (D'Anjou et al. 2008).

\section{Bones}

MRI allows the visualization of changes in the subchondral bone or bone marrow, where the occurrence is associated with insufficiency of the stifle joint. The study conducted by Baird et al. (1998a) demonstrated that using low-field MRI they could visualize focal changes and cyst-like lesions in the subchondral bone in one of six dogs 2 weeks after cranial cruciate ligament insufficiency and in all the subjects investigated after 12 weeks. The changes were round with low signal intensity on T1-weighted images. On inversion recovery and $\mathrm{T} 2$-weighted images they had high 
signal intensity, whereas the peripheral rim had low signal intensity. Changes were localized in the proximal tibial epiphysis (medial tibial condyle, intercondylar eminences) (Baird et al. 1998a). In human medicine it was observed that after acute anterior cruciate ligament rupture in the femur and tibia lesions appeared with high signal intensity. Winegardner et al. (2007) described similar high-signal short tau inversion recovery (STIR) changes in 6 dogs, but their location was a bit different and concerned the intercondylar eminence of the tibia and the intercondylar fossa of the femur. The authors suspected that these invisible anatomical changes could be the source of pain and lameness. It was not observed by Barrett et al. (2009), despite the use of higher field strength devices.

Martig et al. (2007) described the development of changes during the 13 months after the induction of osteoarthritis using the Pond-Nuki model by low-field MRI. They observed changes in the bone marrow of different sizes, variously located and with different signal intensity. The exception concerned the STIR changes in the epiphysis and metaphysis of the tibial eminence, where most cases occurred. However, all changes occurred only in the distal femur and proximal tibia. These abnormalities appeared in various periods of time: lesions on the lateral femoral condyle in the first 4 months, and at the medial from 4 months until the end of the study. Lesions on STIR images had moderate or high signal intensity with a blurred margin. Whereas, on T1-weighted sequences lesions had lower inhomogeneous signal intensity with ill-defined borders, and $\mathrm{T} 2 *$-weighted had the same signal intensity as the adjacent fat marrow. Similar bone marrow abnormalities were observed in all dogs using high-field MRI during the study of the development of osteoarthritis after transection of the cranial cruciate ligament between 1 and 2 months (Nolte-Ernsting et al. 1996), and in another study after six weeks (Libicher et al. 2005). Nolte-Ernsting et al. (1996) described these changes as being in a place with a lower signal intensity than the fat marrow without abnormalities regardless of the sequence used (T1-weighted, fat-suppressed T2-weighted, PD-weighted sequences).

\section{Meniscus}

The canine menisci are crescent-shaped and biconcave fibrocartilaginous structures. Secondary damage to menisci is well described in human and veterinary medicine. The incidence of meniscal lesions associated with cranial cruciate ligament rupture is 48-77.3\% . Most often it affects the medial meniscus and appears as bucket handle tear (Flo and DeYoung 1978, Bennett and May 1991, Baird et al. 1999, Bottcher et al. 2010, Harper et al. 2011). Bennett and May (1991) reported that damage was rarely observed in the lateral meniscus. Moreover, it occurred even when the cranial cruciate ligament was not interrupted (Barrett et al. 2009).

There are several studies describing the effectiveness of magnetic resonance imaging for the detection of meniscal abnormalities associated with cranial cruciate ligament rupture. Particular interest is caused by the difficulty of diagnosing meniscal injury during clinical examination, i.e. using McMurray's test (Corea et al. 1994). The sensitivity of this test in human medicine is low and amounts to only 59\% (Corea et al. 1994). Missing meniscus damage during surgery can cause permanent or recurrent lameness, as well as progression of degenerative changes in the joint. In addition, there is prolonged healing time due to the necessary reoperation for partial or total meniscectomy (Flo 1993, Böttcher et al. 2010).

The image of an intact meniscus is described as a uniformly hypointense triangle on T1-weighted sequences in the dorsal and sagittal planes (Baird et al. 1998a). However, during the anatomic study of the meniscus in the low magnetic field heterogeneous signal increase in the center was observed. The authors supposed that it was due to the gradient echo sequence used and its hyperintense effect (Martig et al. 2006, Blond et al. 2008, Pujol et al. 2011) Similarly, in a high-field study Barrett et al. (2009) defined as an entire meniscus with intrameniscal increased signal intensity that did not reach meniscal margins. In another study using low-field MRI Harper et al. (2011) also recognized meniscal changes which did not extend to the surface as normal, because their clinical importance was not known. It was observed that healing and healed menisci (even more than 6 months after injury) could be characterized by an increased signal which could be mistakenly interpreted as damaged (Arnoczky et al. 1994). Galindo-Zamora et al. (2013) when defining the diagnostic accuracy of high-field MRI, regarded changed menisci as those which characterized by intermeniscal high signal intensity penetrating the surface, or the presence of a complex signal or distortion of meniscal changes.

Martig et al. (2006) proposed a five-point grading system for meniscal lesions in low-field MRI, which was adapted from human medicine. In this system grade 0 lesions are characterized by a meniscus with homogenous low signal intensity, grade 1 and 2 lesions, focal or linear, meant increased intrameniscal signal intensity, respectively. In grade 3 lesions, contrary to 2 grade, change reached the surface of the 
meniscus, which could be observed arthroscopically as a tear. It is the highest grade of meniscal lesion characterized by complex changes of signal. Furthermore, to confirm meniscal lesions they have to be visualized in two different planes (Harper et al. 2011).

Moreover, Martig et al. (2006) investigated the meniscus regularly after transection of the cranial cruciate ligament for a period of 13 months. They reported that during this time all dogs had lesions of the medial meniscus visible on low-field MRI. On the other hand, in high-field MRI meniscal lesions were clearly identifiable 2 weeks after rupture (Galindo-Zamora et al. 2013).

Previously, the authors presented recommended sequences to image meniscus in dogs by high-field MRI as a T2-weighted, proton density-weighted, short tau inversion recovery sequences. Comparison of different sequences used in a high-field MRI study demonstrated that PD-weighted and T2-weighted images had the highest correlations with surgical findings (Barrett et al. 2009). Whereas, in the used low-field MRI the T2-weighted and PD-weighted sequences of good quality images of menisci were obtained, but the STIR sequence did not provide acceptable images. In addition, in images obtained using the device with the strength of $0.2 \mathrm{~T}$, the meniscus was characterized by a lower signal intensity on T2-weighted images than on T1-weighted (McCartney and McGovern 2011). In a low-field MRI study Parizel et al. (1995) reported that using the SE (spin echo) and 3D Fourier transform with steady state precession (FISP) sequences they could visualize a complete tear of the posterior horn of the medial meniscus or foci of intrameniscal abnormalities, because 3D sequences allowed them to obtain more slices (McCartney and McGovern 2011).

In a high-field MRI study Blond et al. (2008) described the detectability of meniscal lesions with a sensitivity of $100 \%$ and a specificity of $94 \%$, but in another study using PD-weighted sequence in the sagittal plane sensitivity was $90 \%$ and specificity was $96 \%$ (Barrett et al. 2009). Whereas Harper et al. (2011), using low-field MRI, successfully detected $90 \%$ of medial meniscus tears. In the second study T2*-weighted GE sequences were used, which allowed an increase in the identification of meniscal lesions, especially histopathological changes. This sequence has a short TE, which improves the SNR ratio, in contrast to T2-weighted sequences (McCartney and McGovern 2011) and improves the contrast between the meniscus and the surrounding tissues (Martig et al. 2006). Galindo-Zamora et al. (2013) has shown that using 3T MRI, pathologic changes of ligaments and menisci could be properly evaluated. Nevertheless, the specificity of the medial meniscus imaging was higher than the sensitivity, and the evaluation of osteoarthritis and damage to cartilage had unsatisfactory accuracy.

Imaging of the caudal horn of the lateral meniscus encounters difficulties associated with interference with the tendon of the popliteus muscle or the meniscofemoral ligament (Smet et al. 1994, Martig et al. 2006, Pujol et al. 2011). This observation was confirmed by Blond et al. (2008) using high-field MRI.

\section{Magnetic resonance arthrography}

Magnetic resonance arthrography is additional possibility to enhance usefulness of MRI, but it is an invasive complement of non-invasive imaging method. Images after high-field MR arthrography had good contrast between ligaments, menisci and cartilage, and allowed the visualization of the popliteal tendon and the meniscal fascicles of the lateral meniscus, which could not be evaluated without a contrast agent. Intra-articular injection of gadolinium obtained increased contrast and high signal-to-noise (Helgason et al. 1997, Banfield and Morrison 2000). Cranial cruciate ligament abnormalities (degenerative changes, partial tears, complete rupture) were better visualized after MR arthrography. Menisci abnormalities, especially complex, oblique tear were also well visualized after MR arthrography, although the diagnostic quality did not differ from that of precontrast images (Banfield and Morrison 2000).

Previously, the low-field MRA study reported that arthrography had no effect on image quality of the normal menisci, but improved the identification of intracapsular ligaments and the joint capsule. However, the authors supposed that MRA may help in diagnosing meniscal injuries (Pujol et al. 2011). Thus far only one publication presented use of low-field MRA in evaluating stifle joint injuries in dogs. Low-field magnetic resonance arthrography allowed the detection of meniscal pathology in only $88 \%$ of cases. There were problems with appropriate visualized meniscal lesions as meniscal cartilage changes, bucket handle tears or axial fringe tears. In the study, the images obtained were confirmed with surgical findings without using arthroscopy, which is considered to be a gold standard (McCartney and McGovern 2012).

\section{Conclusion}

Magnetic resonance imaging is an excellent noninvasive imaging technique, which allows to assess the anatomy of the stifle joint and pathological changes. It 
is a useful method to diagnosis early symptoms of canine stifle osteoarthritis, lesions in the subchondral bone and bone marrow, and in particular ruptured ligaments or lesions of menisci. The image quality and its diagnostic value is dependent on many factors, such as device parameters, imaging planes, sequences and proper patient positioning (Parizel et al. 1995, Rutt and Lee 1996, Marino and Loughin 2010).

High-field MRI allows for obtaining images of canine stifle joint structures of sufficient diagnostic quality (Banfield and Morrison 2000). Previously, it was reported that low-field devices, especially modern ones, allow for obtaining images of a quality equivalent to the images of high-field resonance (Parizel et al. 1995, Baird et al. 1998b, Cotten et al. 2000, Konar and Lang 2011). However, direct extrapolation of the results obtained with high-field to low-field MRI is not recommended (Baird et al. 1999).

With a high-field scanner it is possible to obtain thin slices with a high SNR, which facilitates the detection of subtle changes in menisci even in small dogs (Böttcher et al. 2010). In human medicine, there are few studies of three-dimensional sequence in MRI imaging of the knee (as cited in Kijowski 2010, Naraghi and White 2012). This sequences receive images with relatively thin slices and interslice gaps. Moreover, they improve in-plane spatial resolution, reduce partial volume averaging the effect. Additionally, the advantage of three-dimensional sequences is the ability to post-processing multiplanar reconstruction of images, which allows reformatted images in any imaging plane. This capability has been for a long time a great advantage of CT over MRI (Pretorius and Fishman 1999, Tivers et al. 2009, Naraghi and White 2012). Soler et al. (2007) reported that low-field MRI allowed better evaluation and identification of the stifle join structure than CT. In our opinion, accuracy of three-dimensional sequence to assess stifle joint in dogs should be evaluated.

Previously, authors presented usefulness of fat suppressed T2- and PD-weighted imaging sequences of intra-articular structures of the stifle joint. However, recently it was observed increased use of two-dimensional intermediate-weighted sequence. This sequence was suitable to evaluate all knee joints, due to suitable appropriate tissue contrast and high spatial resolution (Jung et al. 2009, Ristow et al. 2009, Kijowski 2010, Van Dyck et al. 2012). Further study on this sequence to assess the stifle joint in the dog should be performed.

A modification of conventional static MRI is stress magnetic resonance imaging. In a study using high-field MRI, Tremolada et al. (2014) validated this method. They supposed that stress MRI may allow increased accuracy inrecognizing meniscal tears, as it is in human medicine. This leads to the femorotibial joint subluxation of the stifle, which will change the orientation of some intra- and periarticiular structures. An accuracy of this method could be determined.

The magnetic strength of the scanner has a few limitations. In the case of low-field resonance it has a lower signal to noise ratio, and a smaller maximal FOV (field of view) compared with high-field, which affects the image resolution and prolongs the duration of the study (Pujol et al. 2011). Among the disadvantages artifacts can be mentioned, but they appear independent of magnetic strength. However, they differ in terms of type. The images obtained with a low magnetic field are more liable to truncation or partial-volume artifacts, and by means of a high-field chemical shift or more marked metal artifacts (Konar and Lang 2011).

Moreover, the size of the animal and the corresponding size of the joint is an important limiting factor in the study. Martig et al. (2006) showed that the stifle joint of dogs weighing from 7 to $10 \mathrm{~kg}$ could not be evaluated using low-field MRI due to significantly low quality images. Whereas, the investigation using the 3T device allowed for the preparation of good diagnostic quality images of abnormal menisci in the stifle even in dogs weighing $7 \mathrm{~kg}$ (Galindo-Zamora et al. 2013). Nevertheless, even dogs over $20 \mathrm{~kg}$ were selected when designing research using high-field magnetic resonance imaging (D'Anjou et al. 2008, Pujol et al. 2011, Tremolada et al. 2014). Actually, effects of dog size (weight) on imaging of the stifle joint was not determined for low-field and high-field MRI. In our opinion, using low-field MRI, optimal weight is more than $20 \mathrm{~kg}$.

The continuous improvement of magnetic resonance systems, creating new sequences, makes it possible to improve the quality and accuracy of the research (Kegler et al. 2007, as cited in Kijowski 2010). Despite considerable progress and the ever-wider use of MRI in veterinary medicine, imaging of joint disease should be further investigated.

\section{References}

Adamiak Z, Jaskólska M, Matyjasik H, Pomianowski A, Kwiatkowska M (2011) Magnetic resonance imaging of selected limb joints in dogs. Pol J Vet Sci 14: 501-505.

Arnoczky SP, Cooper TG, Stadelmaier DM, Hannafin JA (1994) Magnetic resonance signals in healing menisci: an experimental study in dogs. Arthroscopy 10: 552-557.

Baird DK, Hathcock JT, Kincaid SA, Rumph PF, Kammermann J, Widmer WR, Visco D, Sweet D (1998a) Low-field magnetic resonance imaging of early subchondral cyst-like lesions in induced cranial cruciate ligament deficient dogs. Vet Radiol Ultrasound 39: 167-173. 
Baird DK, Hathcock JT, Rumph PF, Kincaid SA, Visco DM (1998b) Low-field magnetic resonance imaging of the canine stifle joint: normal anatomy. Vet Radiol Ultrasound 39: 87-97.

Baird DK, Kincaid SA, Hathcock JT, Rumph PF, Kammerman J, Visco DM (1999) Effect of hydration on signal intensity of gelatin phantoms using low-field magnetic resonance imaging: possible application in osteoarthritis. Vet Radiol Ultrasound 40: 27-35.

Banfield CM, Morrison WB (2000) Magnetic resonance arthrography of the canine stifle joint: technique and applications in eleven military dogs. Vet Radiol Ultrasound 41: 200-213.

Barrett E, Barr F, Owen M, Bradley K (2009) A retrospective study of the MRI findings in 18 dogs with stifle injuries. J Small Anim Pract 50: 448-455.

Bennett D, May C (1991) Meniscal damage associated with cruciate disease in the dog. J Small Anim Pract 32: 111-117.

Blond L, Thrall DE, Roe SC, Chailleux N, Robertson ID (2008) Diagnostic accuracy of magnetic resonance imaging for meniscal tears in dogs affected with naturally occuring cranial cruciate ligament rupture. Vet Radiol Ultrasound 49: 425-431.

Böttcher P, Brühschwein A, Winkels P, Werner H, Ludewig E, Grevel V, Oechtering G (2010) Value of low-field magnetic resonance imaging in diagnosing meniscal tears in the canine stifle: a prospective study evaluating sensitivity and specificity in naturally occurring cranial cruciate ligament deficiency with arthroscopy as the gold standard. Vet Surg 39: 296-305.

Brandser EA, Riley MA, Berbaum KS, el-Khoury GY, Bennett DL (1996) MR imaging of anterior cruciate ligament injury: independent value of primary and secondary signs. AJR Am J Roentgenol 167: 121-126.

Bredella MA, Tirman PF, Wischer TK, Belzer J, Taylor A, Genant HK (2000) Reactive synovitis of the knee joint: MR imaging appearance with arthroscopic correlation. Skeletal Radiol 29: 577-582.

Carrig CB (1997) Diagnostic imaging of osteoarthritis. Vet Clin North Am Small Anim Pract 27: 777-814.

Conaghan PG, Felson D, Gold G, Lohmander S, Totterman $\mathrm{S}$, Altman R (2006) MRI and non-cartilaginous structures in knee osteoarthritis. Osteoarthritis Cartilage 14 (Suppl A): A87-94.

Corea JR, Moussa M, al Othman A (1994) McMurray's test tested. Knee Surg Sports Traumatol Arthrosc 2: 70-72.

Cotten A, Delfaut E, Demondion X, Lapcgue F, Boukhelifa M, Boutry N, Chastanet P, Gougeon F (2000) MR imaging of the knee at 0.2 and $1.5 \mathrm{~T}$ : correlation with surgery.AJR Am J Roentgenol 174: 1093-1097.

D'Anjou MA, Moreau M, Troncy E, Martel-Pelletier J, Abram F, Raynauld JP, Pelletier JP (2008) Osteophytosis, subchondral bone sclerosis, joint effusion and soft tissue thickening in canine experimental stifle osteoarthritis: comparison between $1.5 \mathrm{~T}$ magnetic resonance imaging and computed radiography. Vet Surg 37: 166-177.

De Smet AA, Tuite MJ, Norris MA, Swan JS (1994) MR diagnosis of meniscal tears: analysis of causes of errors. Am J Roentgenol 163: 1419-1423.

Flo GL (1993) Meniscal injuries. Vet Clin North Am Small Anim Pract 23: 831-843.

Flo GL, DeYoung D (1978) Meniscal injuries and medial meniscectomy in the canine stifle. J Am Anim Hosp Assoc 14: 683-689.
Galindo-Zamora V, Dziallas P, Ludwig DC, Nolte I, Wefstaedt P (2013) Diagnostic accuracy of a short-duration 3 Tesla magnetic resonance protocol for diagnosing stifle joint lesions in dogs with non-traumatic cranial cruciate ligament rupture. BMC Vet Res 9: 40.

Gavin PR (2011) Growth of clinical veterinary magnetic resonance imaging. Vet Radiol Ultrasound 52 (1 Suppl 1) S2-S4.

Harper TA, Jones JC, Saunders GK, Daniel GB, Leroith T, Rossmeissl E (2011) Sensitivity of low-field T2 images for detecting the presence and severity of histopathologic meniscal lesions in dogs. Vet Radiol Ultrasound 52: 428-435.

Hayashi K, Manley PA, Muir P (2004) Cranial cruciate ligament pathophysiology in dogs with cruciate disease: a review. J Am Anim Hosp Assoc 40: 385-390.

Helgason JW, Chandnani VP, Yu JS (1997) MR arthrography: a review of current technique and applications. AJR Am J Roentgenol 168: 1473-1480.

Jung JY, Jee WH, Park MY, Lee SY, Kim JM (2012) Meniscal tear configurations: categorization with 3D isotropic turbo spin-echo MRI compared with conventional MRI at 3 T. AJR Am J Roentgenol 198: W173-180.

Kegler C, Seton HC, Hutchison JM (2007) Prepolarized Fast Spin-Echo Pulse Sequence for Low-Field MRI. Magn Reson Med 57: 1180-1184.

Kijowski R (2010) Three-Dimensional Magnetic Resonance Imaging of Joints. Top Magn Reson Imaging 21: 297-313.

Konar M, Lang J (2011) Pros and cons of low-field magnetic resonance imaging in veterinary practice. Vet Radiol Ultrasound 52 (1 Suppl 1): S5-S14.

Libicher M, Ivancic M, Hoffmann M, Wenz W (2005) Early changes in experimental osteoarthritis using the Pond-Nuki dog model: technical procedure and initial results of in vivo MR imaging. Eur Radiol 15: 390-394.

Marino DJ, Loughin CA (2010) Diagnostic imaging of the canine stifle: a review. Vet Surg 39: 284-295.

Martig S, Boisclair J, Konar M, Spreng D, Lang J (2007) MRI characteristics and histology of bone marrow lesions in dogs with experimentally induced osteoarthritis. Vet Radiol Ultrasound 48: 105-112.

Martig S, Konar M, Schmokel HG, Rytz U, Spreng D, Scheidegger J, Hohl B, Kircher PR, Boisclair J, Lang $\mathrm{J}$ (2006) Low-field Mri and arthroscopy of meniscal lesions in ten dogs with experimentally induced cranial cruciate ligament insufficiency. Vet Radiol Ultrasound 47: 515-522.

McCartney W, McGovern F (2011) Optimising the MRI protocol for imaging of the canine stifle meniscus using a low field system. Intern J Appl Res Vet Med 9: 392-395.

McCartney WT, McGovern F (2012) Use of low-field MRA to presurgically screen for medial meniscus lesions in 30 dogs with cranial cruciate deficient stifles. Vet Rec 171: 47.

Macchia RJ, Termine JE, Buchen CD (2007) Raymond V. Damadian, M.D.: magnetic resonance imaging and the controversy of the 2003 Nobel Prize in Physiology or Medicine. J Urol 178: 783-785.

Mink JH, Reicher MA, Crues JV, Deutsch AL (1993) MRI of the knee. 2nd ed., New York, Raven Press.

Nemec SF, Marlovits S, Trattnig S, Matzek W, Mayerhoefer ME, Krestan CR (2008) High-resolution magnetic resonance imaging and conventional magnetic resonance imag- 
ing on a standard field-strength magnetic resonance system compared to arthroscopy in patients with suspected meniscal tears. Acad Radiol 15: 928-933.

Nolte-Ernsting CC, Adam G, Buhne M, Prescher A, Gunther RW (1996) MRI of degenerative bone marrow lesions in experimental osteoarthritis of canine knee joints. Skeletal Radiol 25: 413-420.

Parizel PM, Dijkstra HA, Geenen GP, Kint PA, Versteylen RJ, van Wiechen PJ, De Schepper AM (1995) Low-field versus high-field MR imaging of the knee: a comparison of signal behaviour and diagnostic performance. Eur J Radiol 19: 132-138.

Podadera J, Gavin P, Saveraid T, Hall E, Chau J, Makara M (2014) Effects of stifle flexion angle and scan plane on visibility of the normal canine cranial cruciate ligament using low-field magnetic resonance imaging. Vet Radiol Ultrasound 55: 407-413.

Pretorius ES, Fishman EK (1999) Spiral CT and three dimensional CT of musculoskeletal pathology. Emergency room applications. Radiol Clin North Am 37: 953-974.

Pujol E, van Bree H, Cauzinille L, Poncet C, Gielen I, Bouvy B (2011) Anatomic study of the canine stifle using low-field magnetic resonance imaging (MRI) and MRI arthrography. Vet Surg 40: 395-401.

Reed AL, Payne JT, Constantinescu GM (1995) Ultrasonographic anatomy of the normal canine stifle. Vet Radiol Ultrasound 36: 315-321.

Ristow O, Steinbach L, Sabo G, Krug R, Huber M, Rauscher I, Ma B, Link TM (2009) Isotropic 3D fast spin-echo imaging versus standard $2 \mathrm{D}$ imaging at $3.0 \mathrm{~T}$ of the knee-image quality and diagnostic performance. Eur Radiol 19: 1263-1272.

Rutt BK, Lee DH (1996) The impact of field strength on image quality in MRI. J Magn Reson Imaging 6: 57-62.

Sabiston CP, Adams ME, Li DK (1987) Magnetic resonance imaging of osteoarthritis: correlation with gross pathology using an experimental model. J Orthop Res 5: $164-172$.

Simpler RE, Kerwin SC, Eichelberger BM, Wall CR, Thompson JA, Padua A, Purdy D, Griffin JF 4th. (2014) Evaluation of the WARP-turbo spin echo sequence for
3 Tesla magnetic resonance imaging of stifle joints in dogs with stainless steel tibial plateau leveling osteotomy implants. Vet Radiol Ultrasound 55: 414-419.

Soler M, Murciano J, Latorre R, Belda E, Rodriguez MJ, Agut A (2007) Ultrasonographic, computed tomographic and magnetic resonance imaging anatomy of the normal canine stifle joint. Vet J 174: 351-361.

Tivers MS, Mahoney PN, Baines SA, Corr SA (2009) Diagnostic accuracy of positive contrast computed tomography arthrography for the detection of injuries to the medial meniscus in dogs with naturally occurring cranial cruciate ligament insufficiency. $\mathbf{J}$ Small Anim Pract 50: 324-332.

Tremolada G, Winter MD, Kim SE, Spreng D, Pozzi A (2014) Validation of stress magnetic resonance imaging of the canine stifle joint with and without an intact cranial cruciate ligament. Am J Vet Res 75: 41-47.

Tsai KJ, Chiang H, Jiang CC (2004) Magnetic resonance imaging of anterior cruciate ligament rupture. BMC Musculoskelet Disord 5: 21.

Van Dyck P, De Smet E, Veryser J, Lambrecht V, Gielen JL, Vanhoenacker FM, Dossche L, Parizel PM (2012) Partial tear of the anterior cruciate ligament of the knee: injury patterns on MR imaging. Knee Surg Sports Traumatol Arthrosc 20: 256-261.

Vahey TN, Broome DR, Kayes KJ, Shelbourne KD (1991) Acute and chronic tears of the anterior cruciate ligament: differential features at MR imaging. Radiology 181: 251-253.

Widmer WR, Buckwalter KA, Braunstein EM, Visco DM, O'Connor BL (1991) Principles of magnetic resonance imaging and application to the stifle joint in dogs. J Am Vet Med Assoc 198: 1914-1922.

Winegardner KR, Scrivani PV, Krotscheck U, Todhunter RJ (2007) Magnetic resonance imaging of subarticular bone marrow lesions in dogs with stifle lameness. Vet Radiol Ultrasound 48: 312-317.

Witsberger TH, Villamil JA, Schultz LG, Hahn AW, Cook JL (2008) Prevalence of and risk factors for hip dysplasia and cranial cruciate ligament deficiency in dogs. J Am Vet Med Assoc 232: 1818-1824. 